\title{
TRIAGEM AUDITIVA NEONATAL: ÍNDICE DE PASSA-FALHA E RELAÇÃO COM AS INTERCORRÊNCIAS GESTACIONAIS E NEONATAIS
}

\author{
Patrícia Arruda de Souza Alcarás, Paula Rodrigues Vicentine \\ Universidade do Oeste Paulista - UNOESTE, Curso de Fonoaudiologia, Presidente Prudente, SP. E-mail: \\ parruda@unoeste.br
}

\section{RESUMO}

O objetivo do estudo foi verificar o índice de passa-falha em neonatos submetidos à triagem auditiva neonatal (TAN) e relacionar essa incidência com as intercorrências gestacionais e neonatais. A amostra foi composta por 55 neonatos, que realizaram a TAN até os 28 dias de vida, nos meses de dezembro de 2014 e janeiro de 2015. A coleta dos dados foi realizada com aplicação de um questionário às mães e pela análise dos resultados do teste das emissões otoacústicas evocadas (EOAE). Os resultados revelaram que a maioria dos neonatos era a termo e apresentou pelo menos uma intercorrência gestacional (54,55\%) ou neonatal (40\%). Quanto aos resultados do teste das EOAE, não houve diferença estatisticamente significativae entre o grupo de neonatos com intercorrências gestacionais ou neonatais com o grupo de neonatos sem intercorrências. Conclui-se que as intercorrências apresentadas não influenciaram no índice de passa-falha na triagem auditiva neonatal.

Palavras-chave: audição, triagem neonatal, emissões otoacústicas, fatores de risco.

\section{NEONATAL AUDITORY SELECTION: PASS-FAIL INDICATION AND RELATION FROM THE NEONATAL AND GESTATIONAL COMPLICATIONS}

\begin{abstract}
The aim of the study was to verify the pass-fail index in the newborns submitted to the newborn hearing screening (TAN) and list this incidence to the gestational and neonatal complications. The sample was composed by 55 newborns, which performed the TAN until 28 days of life in December, 2014 and January, 2015. The data collection was carried out by the application of a questionnaire for the mothers and by the results analysis from the evoked otoacoustic emissions (EOAE). The results showed that the majority of the newborns were at the term and showed at least one gestational complication (54.55\%) or neonatal (40\%). About the EOAE test results, there was no statistically difference among the newborns group with gestational complications to the one group without complications. It follows that the presented complications haven't influenced in the pass- fail index in the newborn hearing screening.
\end{abstract}

Keywords: hearing, newborn screening, otoacoustic emissions, risk factors.

\section{INTRODUÇÃO}

A epidemiologia pode ser descrita como o estudo da distribuição, da frequência e dos determinantes dos estados ou situações relacionadas à saúde em populações específica, a fim de que haja o controle dos problemas de saúde de coletividades humanas ${ }^{1}$.

Todavia, o reconhecimento de determinados problemas de saúde, como no caso de perdas auditivas, dá o direito aos programas de saúde auditiva desenvolver ações de promoção, proteção, diagnóstico, habilitação e reabilitação auditiva, para que possam ser planejados e encaminhados de acordo com as demandas das diversas regiões do país ${ }^{1}$.

A Lei Federal no 12.303 sancionada pelo Presidente da República no dia 2 de Agosto de 2010, define a obrigatoriedade na realização da Triagem Auditiva Neonatal por intermédio das Emissões Otoacústicas Evocadas em todos os neonatos nascidos em hospitais e maternidades, sejam estes públicos ou privados. Ainda, determina que a realização do exame deva ser feita antes da alta hospitalar ${ }^{2}$. Entretanto, essa 
não é a realidade do nosso país, visto que muitos hospitais ainda não realizam a Triagem Auditiva Neonatal em suas dependências.

A obrigatoriedade para a realização da Triagem Auditiva Neonatal se justifica pela importância de se detectar perdas auditivas de forma precoce, visto que alterações mínimas no sistema auditivo podem influenciar no desenvolvimento da linguagem oral da criança.

Estudos retrospectivos apontam que a identificação precoce possibilita a intervenção ainda no "período crítico", pois o processo de maturação do sistema auditivo central ocorrerá durante os primeiro anos de vida ${ }^{3,4}$.

Além disso, a literatura é clara sobre a representação da perda auditiva na população infantil, e por esse motivo, a deteç̧ão precoce favorecerá na redução dos males provocados pela deficiência auditiva, os quais estudos epidemiológicos poderão favorecer na verificação da eficácia dos programas de Triagem Auditiva Neonatal, bem como observar os principais fatores de risco à audição ${ }^{5}$.

Assim, o presente estudo teve por objetivo verificar o índice de passa-falha em neonatos submetidos à triagem auditiva neonatal em uma Unidade Básica de Saúde de uma cidade do interior do Estado de São Paulo e relacionar essa incidência com as intercorrências gestacionais e neonatais.

\section{METODOLOGIA}

O presente estudo de campo e descritivo teve início após aprovação do Comitê de Ética em Pesquisa (CEP-32860414.1.0000.5515) e Comitê Assessor de Pesquisa Institucional (CAPI, n. 2183) da Universidade do Oeste Paulista - UNOESTE.

A amostra foi constituída por 55 neonatos, de ambos os sexos, que vieram realizar a triagem auditiva neonatal até vinte e oito dias de vida (período considerado neonatal), em uma Unidade Básica de Saúde localizada em uma cidade do interior do Estado de São Paulo, nos meses de dezembro de 2014 e janeiro de 2015.

Cada responsável pelo neonato foi convidado a participar do estudo por meio de um Termo de Consentimento Livre e Esclarecido TCLE com informações sobre o estudo. Ao concordar em participar, o TCLE foi preenchido e assinado.

A coleta dos dados foi realizada pela análise dos resultados obtidos no teste das emissões otoacústicas evocadas, sendo este teste realizado pelo profissional fonoaudiólogo responsável pelo atendimento especializado. A coleta e análise dos dados foram realizados conforme abaixo:

$\checkmark$ Seleção de todos os neonatos
triados nos meses de dezembro
de 2014 e janeiro de 2015 .
$\checkmark$ Análise do índice de passa-falha
dos neonatos triados até os vinte
e oito dias vida, ou seja, a
quantidade de neonatos que
foram aprovados no teste e a
quantidade de neonatos que não
foram aprovados no teste de
triagem auditiva.
Análise da correlação dos
neonatos não aprovados com as
principais intercorrências
gestacionais e neonatais, sendo
separados em dois grupos: sem
intercorrências gestacionais e
neonatais (G1) e com
intercorrências gestacionais e/ou
neonatais (G2).

Os resultados obtidos pela coleta dos dados foram apresentados mediante estatística descritiva, em forma de tabelas, mostrando em valores absolutos e relativos dos neonatos pertencentes a cada grupo, bem como pelos resultados da triagem auditiva.

$\mathrm{Na}$ análise estatística inferencial foi utilizado o teste Exato de Fisher para avaliar a relação entre os resultados no teste de triagem com as intercorrências neonatais e gestacionais. Para análise das intercorrências com o sexo do neonato foi aplicado o teste Qui-quadrado. Todas as análises foram realizadas no programa Bioestat 5.3 utilizando o nível de significância de $\mathrm{p}<0,05$.

\section{RESULTADOS}

A coleta dos dados foi realizada em 71 crianças, entretanto, como o critério de inclusão foi à idade limítrofe de 28 dias de vida, sendo este período considerado neonatal, efetivamente participaram do estudo 55 neonatos. Destes, 21 $(38,18 \%)$ eram do sexo feminino e $34(61,82 \%)$ do sexo masculino.

Levando em consideração o sexo do neonato é possível observar que a maioria dos neonatos apresentou pelo menos uma intercorrência gestacional ou neonatal, sendo maior essa ocorrência no sexo masculino. Entretanto, trata-se de uma amostra homogênea, 
ou seja, o número de neonatos do sexo

foram similares, como mostra a Tabela 1.

masculino e feminino com e sem intercorrências

Tabela 1. Caracterização da amostra em relação ao sexo, dos neonatos com e sem intercorrências gestacionais e neonatais.

\begin{tabular}{lcccc}
\hline Sexo & \multicolumn{2}{c}{$\begin{array}{c}\text { Com intercorrências } \\
\text { gestacionais ou neonatais } \\
\text { \% }\end{array}$} & $\begin{array}{r}\text { Sem intercorrências } \\
\text { gestacionais ou neonatais }\end{array}$ \\
& $\mathbf{N}$ & 18,18 & $\mathbf{N}$ & \% \\
\hline Feminino & 10 & 38,18 & 11 & 20,0 \\
Masculino & 21 & 56,36 & 24 & 23,64 \\
Total & 31 & & & 43,64 \\
\hline
\end{tabular}

Valor de $p=0,0975$ (teste do Qui-quadrado)

No que se refere ao período de nascimento, dos 55 neonatos que compuseram a amostra, apenas um nasceu pré-termo, sendo este do sexo masculino (Tabela 2).

Tabela 2. Idade gestacional dos participantes.

\begin{tabular}{lcccccc}
\hline \multirow{2}{*}{$\begin{array}{l}\text { Idade } \\
\text { Gestacional }\end{array}$} & \multicolumn{2}{c}{$\begin{array}{c}\text { Sexo Feminino } \\
(\mathrm{N}=21)\end{array}$} & \multicolumn{2}{c}{$\begin{array}{c}\text { Sexo Masculino } \\
(\mathrm{N}=34)\end{array}$} & \multicolumn{2}{c}{$\begin{array}{c}\text { Total } \\
(\mathrm{N}=55)\end{array}$} \\
\cline { 2 - 7 } & $\mathbf{N}$ & $\%$ & $\mathbf{N}$ & $\%$ & $\mathbf{N}$ & $\%$ \\
\hline A termo & 21 & 100 & 33 & 97,06 & 54 & 98,18 \\
Pré-termo & 0 & 0 & 1 & 2,94 & 1 & 1,82 \\
\hline
\end{tabular}

Dentre os 55 neonatos, 30 (54,55\%)

apresentaram pelo menos uma intercorrência gestacional, como mostra a Tabela 3.

Tabela 3. Intercorrências gestacionais observadas.

\begin{tabular}{lcccccc}
\hline \multirow{2}{*}{$\begin{array}{l}\text { Intercorrências } \\
\text { gestacionais }\end{array}$} & \multicolumn{2}{c}{$\begin{array}{c}\text { Sexo Feminino } \\
(\mathrm{N}=21)\end{array}$} & \multicolumn{2}{c}{$\begin{array}{c}\text { Sexo Masculino } \\
(\mathrm{N}=34)\end{array}$} & \multicolumn{2}{c}{$\begin{array}{c}\text { Total } \\
(\mathrm{N}=55)\end{array}$} \\
\cline { 2 - 7 } & $\mathbf{N}$ & $\mathbf{\%}$ & $\mathbf{N}$ & $\mathbf{\%}$ & $\mathbf{N}$ & $\mathbf{\%}$ \\
\hline Infecção urinária & 7 & 33,33 & 13 & 38,23 & 20 & 36,36 \\
Hipertensão arterial & 6 & 28,57 & 4 & 11,76 & 10 & 18,18 \\
Uso de fumo e álcool & 0 & 0 & 5 & 14,70 & 5 & 9,09 \\
Exposição a raio-x & 0 & 0 & 3 & 8,82 & 3 & 5,45 \\
Diabetes & 2 & 9,52 & 0 & 0 & 2 & 3,63 \\
Herpes & 0 & 0 & 1 & $2,94 \%$ & 1 & 1,81 \\
Uso de psicotrópicos & 0 & 0 & 1 & $2,94 \%$ & 1 & 1,81 \\
\hline
\end{tabular}

Além das intercorrências gestacionais, 22 (40\%) neonatos apresentaram intercorrências neonatais, isoladamente ou associada a duas intercorrências, como mostra a Tabela 4.

Tabela 4. Intercorrências neonatais observadas.

\begin{tabular}{l|c|c|c|c|c|c}
\hline \multirow{2}{*}{$\begin{array}{l}\text { Intercorrências } \\
\text { neonatais }\end{array}$} & \multicolumn{2}{c|}{$\begin{array}{c}\text { Sexo Feminino } \\
(\mathrm{N}=21)\end{array}$} & $\begin{array}{c}\text { Sexo Masculino } \\
(\mathrm{N}=34)\end{array}$ & \multicolumn{2}{c}{$\begin{array}{c}\text { Total } \\
(\mathrm{N}=55)\end{array}$} \\
\cline { 2 - 7 } & $\mathbf{N}$ & $\%$ & $\mathbf{N}$ & $\%$ & $\mathbf{N}$ & $\%$ \\
\hline Hiperbilirrubinemia & 3 & 14,28 & 17 & 50,00 & 20 & 36,36 \\
Consanguinidade & 1 & 4,76 & 2 & 5,88 & 3 & 5,45 \\
\hline
\end{tabular}

Em relação aos resultados das Emissões Otoacústicas Evocadas Transientes (EOAETs), independente das intercorrências apresentadas na gestação ou no período neonatal e levando em consideração o sexo do neonato, a maioria passou (foram aprovados) na triagem auditiva neonatal. Ao relacionar o número de neonatos aprovados e reprovados com o sexo, a análise 
estatística evidenciou não haver diferença

Tabela 5.

estatisticamente significante, como mostra a

Tabela 5. Resultado das Emissões Otoacústicas Evocadas Transientes (EAOETs) em relação ao sexo.

\begin{tabular}{lcccc}
\hline \multirow{2}{*}{ Sexo } & \multicolumn{2}{c}{ Não aprovados } & \multicolumn{2}{c}{ Aprovados } \\
& N & \% & N & $\%$ \\
\hline Feminino & 5 & 9,1 & 16 & 29,1 \\
Masculino & 2 & 3,6 & 32 & 58,2 \\
Total & 7 & 12,7 & 48 & 87,3 \\
\hline
\end{tabular}

Valor de $p=0,0659$ (teste exato de Fisher)

Levando em consideração o grupo de neonatos de gestantes que apresentaram intercorrências no período gestacional, nota-se, também, que a maioria dos neonatos foi aprovada no teste das EOAETs. Ao verificar se a ocorrência de aprovação foi mais significante nos neonatos de gestantes que apresentaram intercorrências, em comparação aos neonatos sem intercorrências, o resultado do teste estatístico evidenciou não haver diferença (Tabela 6).

Tabela 6. Resultado das Emissões Otoacústicas Evocadas Transientes (EAOET) dos neonatos com e sem intercorrência e o sexo.

\begin{tabular}{llcccccc}
\hline $\begin{array}{l}\text { Intercorrências } \\
\text { gestacionais }\end{array}$ & & \multicolumn{2}{c}{$\begin{array}{c}\text { Sexo Feminino } \\
(\mathrm{N}=21)\end{array}$} & $\begin{array}{c}\text { Sexo Masculino } \\
(\mathrm{N}=34)\end{array}$ & \multicolumn{2}{c}{$\begin{array}{c}\text { Total } \\
(\mathrm{N}=55)\end{array}$} \\
\cline { 2 - 8 } & $\mathbf{N}$ & $\mathbf{N}$ & $\mathbf{N}$ & $\mathbf{N}$ & $\mathbf{N}$ & $\%$ \\
\hline $\begin{array}{l}\text { Com } \\
\text { intercorrência }\end{array}$ & Aprovados & 9 & 42,85 & 21 & 61,76 & 30 & 54,54 \\
& Reprovados & 1 & 4,76 & 0 & 0 & 1 & 1,81 \\
\hline $\begin{array}{l}\text { Sem } \\
\text { intercorrência }\end{array}$ & Aprovados & 8 & 38,09 & 11 & 32,35 & 19 & 34,54 \\
& Reprovados & 3 & 14,28 & 2 & 5,88 & 5 & 9,09 \\
\hline
\end{tabular}

Valor de $\mathrm{p}=0,0501$ (teste exato de Fisher)

Quanto ao período do nascimento, a Tabela 7 mostra que o neonato pré-termo passou no teste das EOAETs, sendo que 7 (12,7\%) neonatos a termo não foram aprovados no teste.
Ao relacionar a idade gestacional com a aprovação no teste das EOATs, o teste estatístico evidenciou não haver relação significativa.

Tabela 7. Resultado das Emissões Otoacústicas Evocadas Transientes (EAOET) em relação ao período gestacional.

\begin{tabular}{lcccc}
\hline & \multicolumn{2}{c}{ Não aprovados } & \multicolumn{2}{c}{ Aprovados } \\
& $\mathbf{N}$ & $\mathbf{\%}$ & $\mathbf{N}$ & $\%$ \\
\hline A termo & 7 & 12,7 & 47 & 85,5 \\
Pré-termo & 0 & 0 & 1 & 1,8 \\
Total & 7 & 12,7 & 48 & 87,3 \\
\hline
\end{tabular}

Valor de $p=0,8727$ (teste Exato de Fisher)

Levando em consideração cada intercorrência gestacional, nota-se na Tabela 8 que a maioria dos neonatos passou no teste. Ao relacionar a ocorrência de reprova no teste com a intercorrência gestacional o teste estatístico evidenciou não haver relação significativa. 
Tabela 8. Resultado das Emissões Otoacústicas Evocadas Transientes (EAOET) em relação a cada intercorrência apresentada na gestação.

\begin{tabular}{lcc}
\hline Intercorrência & $\begin{array}{c}\text { Não aprovados } \\
\text { \% }\end{array}$ & $\begin{array}{c}\text { Aprovados } \\
\%\end{array}$ \\
\hline Infeção urinária $(n=20)$ & 9,52 & 90,48 \\
Hipertensão arterial $(n=10)$ & 10,00 & 90,00 \\
Drogas lícitas $(n=5)$ & 0 & 100 \\
Exposição a raio-X $(n=3)$ & 0 & 100 \\
Diabetes gestacional $(n=2)$ & 0 & 100 \\
Psicotrópicos $(n=1)$ & 0 & 100 \\
Herpes $(n=1)$ & 0 & 100 \\
\hline
\end{tabular}

Valor de $p=0,9150$ (teste Extado de Fisher)

Em relação às intercorrências neonatais, apenas um neonato que apresentou histórico de hiperbilirrubinemia não foi aprovado no teste das EOAETs, como mostra a Tabela 9.

Tabela 9. Resultado das Emissões Otoacústicas Evocadas Transientes (EAOET) em relação às intercorrências apresentadas no período neonatal.

\begin{tabular}{lcc}
\hline Intercorrência & $\begin{array}{c}\text { Não aprovados } \\
\%\end{array}$ & $\begin{array}{c}\text { Aprovados } \\
\%\end{array}$ \\
\cline { 2 - 4 } Consanguinidade $(n=3)$ & 0 & 100 \\
Hiperbilirrubinemia $(n=20)$ & 5,00 & 95,00 \\
\hline
\end{tabular}

Valor de $p=0,08696$ (teste Exato de Fisher)

\section{DISCUSSÃO}

Para a efetividade dos programas de Triagem Auditiva Neonatal, estima-se que 95\% dos neonatos nascidos em uma determinada maternidade sejam submetidos ao Teste da Orelhinha ${ }^{6}$.

A população envolvida no presente estudo foram neonatos triados em uma Unidade Básica de Saúde (UBS) Central de um município localizado no interior do Estado de São Paulo, pois até o momento da coleta dos dados, no presente município, não constam de profissionais inseridos nas maternidades para a realização das triagens auditivas.

De acordo com o responsável pelo serviço de atendimento na UBS, os neonatos que recebem alta nas maternidades do município são encaminhados para a realização do Teste do Pezinho e vacinação. No momento em que realizam o Teste do Pezinho, esses neonatos são agendados para a realização do Teste da Orelhinha, sendo este feito em outro momento.

Para que haja menor incidência de evasão, ou seja, de neonatos que recebem alta hospitalar e não que realizam a triagem auditiva neonatal, estudo aponta que é recomendável que a triagem auditiva seja realizada antes da alta hospitalar do recém-nascido, levando em consideração as condições econômicas da população para se locomoverem até o local da realização do exame ${ }^{7}$.

De acordo com o documento do Comitê Multidisciplinar em Saúde Auditiva, mesmo havendo aprovação de leis municipais e estaduais, ainda há diversas maternidades públicas que não realizam o programa de Triagem Auditiva Neonatal Universal (TANU), sistematicamente. No mesmo documento, afirma-se que todos os neonatos deveriam ter acesso à TANU até o primeiro mês de vida ${ }^{5}$.

No município avaliado todos os neonatos nascidos são encaminhados para a realização da triagem antes do primeiro mês de vida, porém, sabe-se que pode haver ocorrência de evasão, uma vez que não é realizada na própria maternidade e antes da alta hospitalar.

Deve-se levar em consideração que o município está realizando o programa de triagem auditiva de forma precoce, pois segundo as recomendações do Joint Committe Infant Hearing, crianças com idade em até um mês de vida devem passar pela triagem, independente destas apresentarem ou não risco para perda auditiva ${ }^{6}$.

Quanto ao exame aplicado para a realização da triagem auditiva, o local realiza somente o teste das emissões otoacústicas evocadas, como prevê a Lei no 12.303 sancionada pelo Presidente da República no dia 2 de agosto 
de $2010^{2}$. No entanto, estudos apontam a necessidade da realização dos Potenciais Evocados Auditivos de Tronco Encefálico Automático (PEATE-A) em neonatos que vierem apresentar indicadores de risco para perda auditiva, uma vez que também são considerados de risco para perdas auditivas retrococleares ${ }^{5,7}$.

Dos 55 neonatos que compuseram a amostra, 54 (98,18\%) eram a termo, ou seja, apresentaram idade gestacional superior a 36 semanas (Tabela 2). Em relação às intercorrências gestacionais, $54,55 \%$ dos neonatos apresentaram pelo menos uma intercorrência, como mostrou a Tabela 3.

A literatura evidencia que crianças com histórico familiar de perda auditiva na infância, com permanência em UTIN por mais de cinco dias e que necessitaram fazer uso de oxigenação por membrana extra-corporal, ventilação mecânica, uso de medicações ototóxicas ou diuréticos de alça, com histórico de Hiperbilirrubinemia com exsanguíneo transfusão, infecções congênitas, que apresentarem anomalias craniofaciais, síndromes associadas à perdas auditivas, ou que tenham apresentado infecções pós-natais, são consideradas de risco para perda auditiva ${ }^{5,6}$.

Além disso, autores apontam outros fatores de risco, sendo eles: boletim do apgar de 0 a 4 no primeiro minuto e 0 a 6 no quinto minuto, permanência na incubadora por mais de sete dias, consanguinidade entre os pais, peso inferior ao nascimento a $1500 \mathrm{~g}$, pequena para idade gestacional, histórico de hemorragia ventricular, convulsões neonatais, alcoolismo materno ou uso de drogas psicotrópicas na gestação, também são considerados de risco para perda auditiva ${ }^{8-10}$.

Dentre as intercorrências gestacionais a infecção de urinária foi a mais relatada pelos pais e/ou responsáveis dos neonatos (36,36\%), como mostrou a Tabela 3. Declara-se que todas as mães que apresentaram infecção urinária na gestação fizeram uso de antibioticoterapia.

O simples fato de a gestante apresentar infecção urinária não classifica o neonato de risco para perda auditiva, mas sim, pelo uso de antibióticos para o tratamento da infecção.

A diminuição da capacidade renal em armazenar a urina durante a gravidez reduz a atividade antibacteriana, passando a excretar menor quantidade de potássio e maior quantidade de glicose e aminoácidos, além de substâncias de degradação hormonal, favorecendo a proliferação bacteriana. Autores referiram que a urina da gestante apresenta $\mathrm{pH}$ mais alcalino, propiciando o crescimento das bactérias presentes no trato urinário ${ }^{11}$.

Ao realizar um estudo em 2010, autores relataram é comum à infecção urinária em mulheres jovens, sendo considerada uma das complicações clínicas mais frequentes na gestação, pois neste período ocorrem diversas modificações, principalmente mediadas pelas alterações hormonais, o que pode favorecer o aparecimento de infeç̧ões urinárias. Como o aumento da produção da urina pode levar a ocorrência de bactérias e infecções, o tratamento clínico se dá pelo uso de antibióticos. Dentre eles, encontra-se a ampicilina, medicamento considerado de risco para perda auditiva. Com isso, os autores relataram que neonatos de gestantes que apresentaram histórico de infecção urinária podem ser considerados de risco para perda auditiva mediante ao tratamento clínico ${ }^{12}$.

Além da infecção urinária, a presença da hipertensão arterial também foi bastante relatada pelas mães e/ou responsáveis pelos neonatos $(18,18 \%)$.

Autores relataram em seu estudo alta prevalência de risco materno decorrente da hipertensão arterial. Segundo eles, mães com idade mais avançada apresentam maiores riscos de hipertensão arterial $^{13}$.

Em outro estudo autores apontam que a hipertensão gestacional eleva os riscos prénatais, pois pode favorecer no baixo peso dos neonatos ao nascimento, bem como na prematuridade, sendo estes uns dos indicadores de risco para perda auditiva ${ }^{14}$.

O uso de álcool e fumo na gestação também pode ser considerado de riscos para perda auditiva em neonatos. $O$ uso de álcool está ligado aos efeitos teratogênicos, levando à criança apresentar síndrome alcoólica fetal (SAF). Dentre as manifestações, crianças que nascem com SAF podem apresentar anomalias craniofaciais, déficit no crescimento, alterações no sistema nervoso central e diversas malformações associadas, elevando o risco de alterações auditivas ${ }^{12}$.

Quanto ao uso de fumo, os autores relataram haver relação entre o baixo peso ao nascimento, ocorrência de parto prematuro e malformações congênitas, como a presença de fissuras labiopalatais e distúrbios do sistema nervoso central. Além disso, o monóxido de carbono presente no fumo altera o suporte de oxigênio para o feto, podendo causar hipóxia ${ }^{12}$. 
Tais manifestações favorecem o aparecimento de perdas auditivas em neonatos.

Dentre as intercorrências neonatais, a hiperbilirrubinemia foi a mais citada pelas mães e/ou responsáveis dos neonatos triados $(36,36 \%)$, entretanto, nenhuma foi ao nível de necessitar realizar a exsanguíneotransfusão.

A literatura aponta que a hiperbilirrubinemia neonatal é destacada como indicador de risco para a ocorrência da neuropatia auditiva/dessincronia auditiva somente se esta for a nível exsanguíneotransfusão, visto que níveis de bilirrubina indireta elevados durante o período neonatal alteram o sistema nervoso central, alcançando as estruturas mais internas do sistema auditivo ${ }^{5,15}$.

Ao relacionar os resultados do teste das Emissões Otoacústicas Evocadas Transientes (EOAETs) com o sexo do neonato, independente do sexo a maioria foi aprovada no teste $(87,3 \%)$, não havendo diferença significante quanto a essa variável.

A literatura evidencia que a variável sexo é um dos fatores que pode interferir nos resultados da triagem auditiva. Com intuito de verificar o índice de passa/falha na triagem auditiva neonatal em neonatos sem risco para perda auditiva, e levando em consideração as variáveis: sexo, tipo de parto e tempo de vida, autores verificaram maior ocorrência de reprova no sexo masculino, porém este resultado não foi estatisticamente significante ao comparar com o sexo feminino ${ }^{16}$.

Quanto aos resultados do teste das EOAETs entre os neonatos que apresentaram intercorrências gestacionais, não houve relação estatisticamente significante entre àqueles que não apresentaram intercorrências $(p=0,0501)$, ou seja, a ocorrência de falha no teste foi similar entre os dois grupos. A mesma observação pode ser feita em relação ao período de concepção (pré-termo e a termo).

A literatura é unânime quanto aos indicadores de risco para perda auditiva e incidência de perda auditiva na população neonatal, sendo essa incidência maior em neonatos considerados de risco para perda auditiva ${ }^{5}$.

Estima-se que a incidência de perda auditiva em neonato seja de 1 a 6 para cada 1000 nascimentos, e de 1 a 50 para cada 1000 nascimentos em neonatos considerados de risco $^{17}$.
Nem todas as intercorrências apresentadas pelos neonatos no período gestacional ou neonatal (até 28 dias de vida) são consideradas de riscos para perda auditiva. Entretanto, fazer a relação destas intercorrências com o índice de passa e falha é importante para compreender se essas intercorrências podem influenciar no resultado da triagem auditiva neonatal.

Assim, independente da intercorrência apresentada pelo neonato, a triagem auditiva deve ser feita de forma universal, ou seja, em pelo menos $95 \%$ dos neonatos. Além disso, não basta realizar a triagem auditiva, é necessário dar seguimento com o acompanhamento fonoaudiológico em caso de falha, ou realizar outros métodos de avaliação em neonatos que suspeitarem de alterações retrococleares e/ou centrais. 0 acompanhamento do desenvolvimento da função auditiva é relevante nos primeiros anos de vida.

As leis citadas no presente estudo servem para promover ao neonato o reconhecimento precoce da perda auditiva, e também, a possível adaptação do Aparelho de Amplificação Sonora Individual (AASI), para que assim não haja degradações no desenvolvimento linguístico da criança.

\section{CONCLUSÃO}

Diante dos resultados apresentados é possível concluir que: a maioria dos neonatos era a termo e apresentou pelo menos uma intercorrência gestacional ou neonatal. Entretanto, a maioria foi aprovada no teste das emissões otoacústicas evocadas.

Pode-se observar que as intercorrências apresentadas pelos neonatos não tiveram relação estatisticamente significantes com o resultado de triagem auditiva, pois as intercorrências não influenciaram no resultado. Vale ressaltar que o tamanho da amostra foi restrita e, assim, recomenda-se a realização de novos estudos com uma amostra maior.

\section{CONFLITOS DE INTERESSE}

Os autores declaram não haver qualquer potencial de conflito de interesse que possa interferir na imparcialidade deste trabalho científico. 


\section{REFERÊNCIAS}

1. Silva DTC, Lewis DR. Epidemiologia descritiva da deficiência auditiva na infância. Distúrb Comum. 2013;25(1):148-51.

2. Brasil. Presidência da República, Subchefia para Assuntos Jurídicos. Lei no 12.303 de 2 de agosto de 2010. Dispõe sobre a obrigatoriedade de realização do exame denominado Emissões Otoacústicas Evocadas [Internet]. Brasília, DF; 2010. [acesso em 2015 nov. 10]. Disponível em: http://www.planalto.gov.br/ccivil 03/ Ato20072010/2010/Lei/L12303.htm

3. Durante A L, Carvallo RMM, Costa FS, Soares JC. Características das emissões otoacústicas por transientes em programa de triagem auditiva neonatal. Pró-Fono Rev Atual Cient. 2005;17(2):133-40. DOI:

http://dx.doi.org/10.1590/S0104-

$\underline{56872005000200002}$

4. Borges CAB, Moreira LMO, Pena GM, Fernandes FR, Borges BCB, Otani BH. Triagem auditiva neonatal universal. Rev Arq Int Otorrinolaringol. 2006;10(1):28-34.

5. Lewis DR, Marone SAM, Mendes BCA, Cruz OLM, Nóbrega M. Comitê Multidisciplinar em Saúde Auditiva - COMUSA. Braz J Otorhinolaryngol. 2010;76(1):121-8.

6. Joint committee on infant hearing $(\mathrm{JClH})$. Position statement: principles and guidelines for early hearing detection and intervention programs. Pediatrics. 2007;120(4):898-921. DOI: https://doi.org/10.1542/peds.2007-2333

7. Freitas VS. Especificidade e taxa de falsopositivo em três protocolos de triagem auditiva neonatal [Dissertação]. Bauru: Faculdade de Odontologia de Bauru, Universidade de São Paulo; 2006.2 DOI: https://doi.org/10.11606/D.25.2006.tde10092007-165438

8. Pereira PKS, Martins AS, Vieira MR, Azevedo MF. Programa de triagem auditiva neonatal: associação entre perda auditiva e fatores de risco. Pró-Fono Rev Atual Cient. 2007;19(3):26778. DOI: http://dx.doi.org/10.1590/S010456872007000300005

9. Vieira EP, Miranda EC, Azevedo MF, Garcia MV. Ocorrência dos indicadores de risco para a deficiência auditiva infantil no decorrer de quatro anos em um programa de triagem auditiva neonatal de um hospital público. Rev Soc Bras Fonoaudiol. 2007; 12(3):214-20. DOI: http://dx.doi.org/10.1590/S1516$\underline{80342007000300009}$
10. Amado BCT, Almeida EOC, Berni PS. Prevalência de indicadores de risco para surdez em neonatos em uma maternidade paulista. Rev CEFAC. 2009;11(1):18-23. DOI: http://dx.doi.org/10.1590/S151618462009005000020

11. Duarte G, Marcolin AC, Quintana SM, Cavalli RC. Infecção Urinária na gravidez. Rev Bras Ginecol Obstet. 2008;30(2):93-100. DOI: http://dx.doi.org/10.1590/S0100-

\section{8}

12. Griz SMS, Silva ARA, Barbosa CP, Menezes DC, Curado NRPV, Silveira AK et. al. Indicadores de risco para perda auditiva em neonatos e lactentes atendidos em um programa de triagem auditiva neonatal. Rev CEFAC. 2011;13(2):281-90. DOI: http://dx.doi.org/10.1590/S151618462010005000071

13. Ruggieri MM, Lichtig I, Marone SAM. Recémnascidos gerados por mães com alto risco gestacional: estudo das emissões otoacústicas produtos de distorção e do comportamento auditivo. Rev Bras Otorrinolaringol. 2002;68(2):230-7. DOI: http://dx.doi.org/10.1590/S003472992002000200012

14. Chaim SRP, Oliveira SMJV, Kimura AF. Hipertensão arterial na gestação e condições neonatais ao nascimento. Acta Paul Enferm. 2008;21(1):53-8.

DOI:

http://dx.doi.org/10.1590/S0103-

\section{8}

15. Martinho AC, Lewis DR. Achados audiológicos em crianças com Hiperbilirrubinemia neonatal: um enfoque na neuropatia auditiva/ dessincronia auditiva. Rev Distúrbios Comunicação. 2005; 12(2):183-190.

16. Michelon $F$, Rockenbach SP, Floriano $M$, Delgado SE, Barba MC. Triagem auditiva neonatal: índice de passa/falha com relação a sexo, tipo de parto e tempo de vida. Rev CEFAC. 2013;15(5):189-95.

DOI:

http://dx.doi.org/10.1590/S1516$\underline{18462013000500016}$

17. Rezende MCL, Dias FAM. Programa de triagem auditiva neonatal e os fatores de risco para deficiência auditiva. Rev CEFAC. 2009;19(3):267-78.

Recebido para publicação em 29/11/2015

Revisado em 22/09/2016

Aceito em 15/11/2017 